\title{
INCORPORACIÓN DEL MODELO DE LANDAU AL MODELO FRACTAL DE UNA ERUPCIÓN
}

\author{
INCORPORATION OF LANDAU MODEL TO THE FRACTALMODEL OFAN ERUPTION
}

\author{
José Brenes-André \\ Red Ciudadana de Estaciones Meteorológicas \\ Apdo. 290-3015 Costa Rica \\ jbrenes54@gmail.com
}

(Recibido: 26/03/2013; aceptado: 29/01/2016)

\begin{abstract}
The Sequential Fragmentation/Transport model (SFT) is based on a macroscopic level sequential fragmentation. In Brenes \& Alvarado (2013) a fractal model of an eruption is proposed based on SFT, systematizing the process via the inclusion of Hurst Coefficient. Later on, Balankin fractal fracture model is used to include microscopic level fractal fracture processes (Brenes, 2014). In the present work, Landau model for second order transformations is included, and Griffith fracture model and Eötvös rule are deduced, giving rise to by-products mathematical expressions that suggest the existence of fracture processes such as stress corrosion, dilatancy hardening, and vitrification. Lastly, some mathematical results that suggest a glass transition in magma are presented.

Keywords: Balankin, Landau, Brown, Griffith, Sequential fragmentation/transport, SFT.

RESUMEN: El modelo Sequential Fragmentation/Transport (SFT, por su sigla en Inglés) se basa en una fragmentación secuencial a nivel macroscópico. En Brenes \& Alvarado (2013) dicho modelo sirvió de base para proponer un modelo fractal de una erupción, al sistematizar todo el proceso con la inclusión del coeficiente H de Hurst. Esta sistematización fue posteriormente ampliada en Brenes (2014), con ayuda del modelo fractal de fractura propuesto por Balankin, para incluir procesos de fractura a nivel microscópico. En el presente trabajo, la inclusión del modelo de Landau para transformaciones de segundo orden permite deducir el modelo de fractura de Giffith y la regla de Eötvös, y obtener como sub-producto expresiones que sugieren la existencia de procesos de fractura tales como la corrosión por esfuerzos, endurecimiento por dilatación y la vitrificación. Finalmente, se presentan resultados sugerentes de una transición al vidrio en el magma.

Palabras clave: Balankin, Landau, Griffith, Transición del vidrio, Fragmentación secuencial, SFT.
\end{abstract}




\section{INTRODUCCIÓN}

En Brenes (2013) se presentó la aplicación de la SFT a un volcán centroamericano: el Irazú (Costa Rica), incluyéndose la sugerencia de estudiar por separado cada uno de esos mecanismos eruptivos de balística, oleadas, saltación y suspensión, aplicando los planteamientos de Brown (1989) y Wohletz et al. (1989) cuando la dispersión es negativa. En un trabajo posterior (Brenes \& Alvarado, 2013) se complementó ese análisis sistematizando los casos con dispersión positiva, proponiéndose que no es un producto de agregación de partículas en vuelo, sino que corresponden a una fragmentación secundaria a la que se le pudo asignar una segunda dimensión fractal. Se incluyó asimismo una manera de calcular el coeficiente de Hurst asociado a cada sub-población. Lo anterior permitió deducir los límites experimentales que Wohletz et al. (1989) encontraron entre la actividad magmática y la freatomagmática, proponiéndose por primera vez un modelo fractal de una erupción. Recientemente, en Brenes (2014) el modelo fractal de una erupción se amplió para incluir las propuestas ab initio de Balankin (1996, 1997). De esta manera, a partir de consideraciones microscópicas, se pudo dar una explicación geométrica a los dos valores universales del coeficiente de Hurst para fractura. En este trabajo esos resultados se amplían utilizando el modelo de Landau de transformaciones de segundo orden, para deducir el modelo de Griffith, y los tamaños límite deducidos por Balankin (1996, 1997). Igualmente, se muestra como efectos como la corrosión por esfuerzos (stress corrotion), y dilatancia se deducen naturalmente de esta ampliación, con lo que de manera segura el parámetro de dispersión $\gamma$ propuesto por Brown puede ahora considerarse como un parámetro de orden en el proceso de fractura.

El trabajo consta de 8 partes: a) Una breve descripción del modelo de Landau para transformaciones de segundo orden; b) una rederivación de la ecuación de Griffith a partir de lo encontrado en el punto anterior; c) una demostración de que ambos resultados son equivalentes; d) la incorporación explícita de la temperatura; e) un breve análisis de cómo estos resultados se pueden aplicar a materiales dúctiles; f) un breve análisis de todas las posibles opciones que resultan del punto anterior; g) reflexiones sobre la ecuación cúbica de Landau y h) algunas consideraciones de como se podría incluir el proceso de Transición del vidrio.

\section{MODELODE LANDAU PARA TRANSFORMACIONESDESEGUNDOORDEN}

En este trabajo el proceso de fractura se considera como un fenómeno crítico (Guarino et al., 1998), el que se analizará con ayuda del Modelo de Landau para transformaciones de segundo orden. Se refiere al lector al libro Landau \& Lifschitz (1958) para profundizar sobre el tema.

Aquí se darán solo los puntos básicos. A nivel geológico, un cuerpo puede presentarse en diferentes fases: sólida, líquida o gaseosa. La transición de una a otra, al cambiar factores externos como la presión o la temperatura, se le conoce como una transformación de fase de primer orden, y conlleva asociada un calor de fusión, evaporación o de sublimación, según el caso. Las transformaciones de segundo orden se dan cuando el material, sin cambiar su fase sólida, por ejemplo, puede adoptar diferentes maneras de ordenar sus moléculas, cambiando la simetría interna como sucede con el fósforo banco y el rojo.

La teoría de Landau es fenomenológica y tiene que ver básicamente con la simetría del material estudiado, y con las transformaciones de fase causadas por un correspondiente cambio de simetría.

El modelo teórico aceptado para tratar ese tipo de transformación es conocido como el Modelo de Landau, que requiere llevar a cabo los siguientes pasos: a) definir un parámetro de orden, que sería alguna propiedad o combinación de propiedades, tal que es cero en la fase desordenada, y diferente de cero en la fase ordenada. Debe corresponder a una característica interna del sistema que no pueda cambiarse desde el exterior y poderse variar continuamente. No existe ninguna regla en cómo lograr este objetivo; b) escribir la energía libre de Gibbs, G, del sistema como una expansión polinómica finita del parámetro 
de orden, que cumpla con todas las simetrías del sistema (rotacional, traslacional, y cualquier otro grado de libertad interno); c) la dependencia en temperatura, o presión se incluye exclusivamente en el coeficiente del parámetro de menor exponente, dejando los otros constantes.

La idea central es que el sistema puede presentar una forma ordenada (puede determinarse que hay partes del sistema que se distinguen de otras partes), que van a estar asociadas a un parámetro de orden diferente de cero para temperaturas $\mathrm{T}<\mathrm{T}_{\mathrm{C}}$; o presentar una forma carente de cualquier orden (homogénea) para $\mathrm{T}>\mathrm{T}_{\mathrm{C}}$. Tal carencia de orden se define haciendo el parámetro de orden idénticamente cero.

\section{Escogencia del parámetro de orden}

El parámetro $\gamma$ fue introducido en Brown (1989), como la dispersión de la distribución en tamaño de las fracturas, y fue asociada en Wholetz et al. (1989) a una dimensión fractal $\mathrm{D}=-3 \gamma$ para el caso $\gamma<0$. En Brenes (2012), su uso se amplió para obtener 5 relaciones lineales asociadas a cada uno de los procesos de fragmentación. En Brenes \& Alvarado (2012), los casos de $\gamma>0$ se asociaron a fragmentaciones secundarias vía una nueva dimensión fractal $\mathrm{D}=3(1+\gamma)$, que permitió definir un coeficiente de Hurst $(H=1-|\gamma|)$. De esta manera se pudo dar sustento teórico al límite experimental de $\gamma=-1 / 2$ entre productos freatomagmáticos y magmáticos obtenido experimentalmente (Wholetz et al., 1989). Permitió también discriminar entre magma explosivo y magma efusivo; y en el caso del Irazú detectar un posible caso de mezcla de magmas ocurrido en el evento de 1723. En Brenes (2014), con ayuda del modelo de Balankin (1998), la variable $\gamma$ es usada para explicar teóricamente los dos valores universales de $\mathrm{H}$ asociados a procesos de fractura.

En Balankin (1998), se analiza la fractura como un proceso fractal auto afín, y se proponen dos exponentes diferentes para el factor de intensidad de esfuerzos en el ámbito de $1 \mu \mathrm{m}$ a $1 \mathrm{~cm}$. Las vesículas, asociadas a valores positivos de $\gamma$ (Brenes \& Alvarado, 2013), también están en este ámbito (Sparks, 1978). Esta escala mesoscópica es lo suficientemente pequeña para observar como continuas las variaciones de los dominios cristalinos, pero lo suficientemente grande como para que hayan suficientes moléculas para que el parámetro de orden tenga, aunque aproximadamente, un significado termodinámico. Dado que Wholetz et al. (1989) aplicó el parámetro $\gamma$ a granulometrías en ese mismo ámbito, en este trabajo se propone la dispersión $\gamma$ como el parámetro de orden a utilizarse en el modelo de Landau para transformaciones de segundo orden.

El coeficiente de Hurst H también queda definido en ese ámbito, como lo expresa Balankin (1997) al indicar que $\mathrm{H}$ no es otra cosa que el exponente característico del régimen intermedio, y que solo podemos asociar propiedades fractales a una fractura en el ámbito en que la fractura es auto-afin.

\section{Construcción de la energía de Gibbs}

Balankin (1998), a partir de consideraciones $a b$ initio basadas en el carácter fractal de las fracturas, propone como exponentes del factor de intensidad de esfuerzos las igualdades $\alpha=(\mathrm{dH}-\mathrm{d}$ $+1) / 2 \mathrm{H}$ y $\beta=(2 \mathrm{H}-1) / 2 \mathrm{H}$, siendo $\mathrm{H}$ el coeficiente de Hurst asociado a la fractura, y d la dimensión topológica del espacio correspondiente. Brenes (2014) propuso cambiar la dimensión topológica $\mathrm{d}$ por las dos dimensiones fractales $\mathrm{D}=-3 \gamma$ y $\mathrm{D}$ $=3(1+\gamma)$, lo que permite expresar los exponentes para el ámbito $\gamma<0$ como $\alpha^{-}=\left[1-3 \gamma^{2}\right] / 2(1+\gamma)$ y $\beta^{-}=[1+2 \gamma] / 2(1+\gamma)$, y para el ámbito $\gamma>0$ como $\alpha^{+}=[1-3 \gamma-3 \gamma 2] / 2(1-\gamma) ; \mathrm{y} \beta^{+}=[1-2 \gamma] / 2(1-\gamma)$. Los dos valores universales de $\mathrm{H}$ asociados a fracturas se obtienen haciendo cero las expresiones de $\alpha$ y $\beta^{-}$, lo que equivale a estados de equilibrio. Termodinámicamente corresponden a estados en que la energía libre de Gibbs, G, es mínima.

Como punto de partida, en este trabajo se propone que la derivada de $\mathrm{G}$ respecto al parámetro de orden, para el caso del exponente $\alpha$, puede escribirse como:

$$
\begin{aligned}
& \mathrm{dG} / \mathrm{d} \gamma=\mathrm{Q}\left[1-3 \gamma^{2}\right] \gamma /(1+\gamma) \\
& \mathrm{dG} / \mathrm{d} \gamma=\mathrm{Q}\left[1-3 \gamma-3 \gamma^{2}\right] \gamma /(1-\gamma)
\end{aligned}
$$


Donde el coeficiente Q tiene unidades de energía. La expresión (1) corresponde al caso de dispersión negativa, y la (2) a dispersiones positivas. El término $\gamma$ extra se incorporó para lograr que la energía libre $G$ para $\gamma=0$ sea mínima cuando $\mathrm{T}>\mathrm{T}_{\mathrm{C}}$, y mínima para $\gamma \neq 0$ cuando $\mathrm{T}<\mathrm{T}_{\mathrm{C}}$, según lo requiere el modelo de Landau. El término $\mathrm{T}_{\mathrm{C}}$, siguiendo a Landau, corresponde a una temperatura crítica.

Para el caso del exponente $\beta$, debido a que $\mathrm{H}$ es simétrica respecto a $\gamma$, y no involucra ninguna dimensión fractal, proponemos la expresión exacta

$$
\mathrm{dG} / \mathrm{d} \gamma=\mathrm{Q}(1-2|\gamma|) \gamma /(1-|\gamma|)
$$

De esta manera se está incluyendo el principio de von Neumann de que todos los modos compatibles con la simetría deben estar presentes en la distorsión total, aunque no necesariamente con el mismo peso.

\section{Energía de Gibbs exacta}

Efectuando la integración de las ecuaciones (1), (2) y (3a) se obtiene

$$
\begin{aligned}
& \mathrm{G}_{\alpha-}=\mathrm{Q}\left(3 \gamma^{2}-4 \gamma+4 \operatorname{Ln}(1+\gamma)-2 \gamma^{3}-9\right) \\
& \mathrm{G}_{\alpha+}=\mathrm{Q}\left(3 \gamma^{2}+5 \gamma+5 \operatorname{Ln}(1-\gamma)+\gamma^{3}-9\right) \\
& \mathrm{G}_{\beta-}=\mathrm{Q}\left(3 \gamma^{2}+3 \gamma+3 \operatorname{Ln}(1-\gamma)-6\right) \\
& \mathrm{G}_{\beta+}=\mathrm{Q}\left(3 \gamma^{2}-3 \gamma+3 \operatorname{Ln}(1+\gamma)-6\right)
\end{aligned}
$$

La energía de Gibbs para el caso Beta es simétrica alrededor de $\gamma=0$ pues el término $\gamma+$ $\operatorname{Ln}(1-\gamma)$, válido para $\gamma<0$ es idéntico a $-\gamma+\operatorname{Ln}(1$ $+\gamma$ ), válido para $\gamma>0$. Para el caso del exponente Alfa ni la energía de Gibbs ni su primera derivada presentan una discontinuidad en $\gamma=0$.

Aunque las expresiones tienen orígenes diferentes, es evidente que hay un patrón. El término $3 \gamma^{2}$ es común y se asocia a la energía superficial que resulta de la creación de la fractura. El término $\gamma+\operatorname{Ln}(1-\gamma)$ es común a todos, $\mathrm{y}$ se asocia a la energía elástica. Su peso relativo varía de un 3 para el caso de partículas lo suficientemente grandes como para que el esfuerzo en los extremos de la fractura se anule antes de llegar a la superficie de la partícula, pasando a 4 cuando las partículas no son así de grandes, hasta llegar a un 5 cuando, además de su pequeño tamaño, la fractura tiene gas a presión (o sea, una vesícula). La corrección $\gamma 3$ aparece únicamente para el exponente $\alpha$, o sea, para partículas pequeñas, y se asocia a la deformación de la partícula.

\section{Energía de Gibbs aproximada}

En lo que sigue se proponen expresiones aproximadas de la energía de Gibbs para los 3 casos, obtenidas usando solo el numerador de las ecuaciones (1) y (2). La correspondiente para el exponente beta es

$$
\mathrm{dG} / \mathrm{d} \gamma=\mathrm{Q}[1+2 \gamma][1-2 \gamma] \gamma
$$

Como se verá más adelante, no solo los resultados son muy cercanos a las soluciones exactas, sino que permiten deducciones muy esclarecedoras del proceso de fractura.

Definiendo $\mathrm{G}(\gamma=0)=0$ como el nivel de referencia, se obtiene:

$$
\begin{aligned}
& \mathrm{G}-=\mathrm{Q}-\left[\gamma^{2} / 2-3 \gamma^{4} / 4\right] \\
& \mathrm{G}+=\mathrm{Q}+\left[\gamma^{2} / 2-\gamma^{3}-3 \gamma^{4} / 4\right] \\
& \text { para el exponente } \alpha, \mathrm{y} \\
& \mathrm{G}_{\beta}=\mathrm{Q}_{\beta}\left[\gamma^{2} / 2-\gamma^{4}\right]
\end{aligned}
$$

para el exponente $\beta$.

Por la simetría $\gamma \rightarrow-\gamma$ en la ecuación (7), los procesos de fractura en que el exponente $\beta$ sea aplicable, no permite distinguir entre procesos de fractura primarios $(\gamma \leq 0)$ y secundarios $(\gamma \geq 0)$.

En aquellos casos en que deba utilizarse el exponente $\alpha$, hay un rompimiento natural de simetría, pues la ecuación (5) solo es válida para $\gamma$ $\leq 0$, en tanto que la ecuación (6) aplica solo para $\gamma$ $\geq 0$, proceso conoce se conoce como rompimiento de simetría en el modelo de Landau.

Para garantizar que los 3 gráficos asociados tengan la forma esperada según Landau se 
escogerá $\mathrm{Q}<0$. Esta forma esperada está definida por los signos de cada uno de sus términos, que al estar determinados por consideraciones físicas fundamentales, en nuestro caso se espera que las ecuaciones (5), (6) y (7) reflejen otros procesos físicamente viables.

A pesar de originarse en expresiones muy diferentes, llama la atención la similitud de las 3 expresiones de la energía de Gibbs. Las ecuaciones (5) y (7) contienen solo potencias pares, que en el modelo de Landau es algo impuesto por la simetría esperada en el proceso. En nuestro caso, refleja la simetría Z2 (invariancia frente a rotación de $180^{\circ}$ ) de las fracturas. La ecuación (6) difiere de la (5) por el término $\gamma 3$ extra, usualmente asociado a fluctuaciones del parámetro de orden alrededor de su valor promedio y a un cambio de fase de primer orden, una posibilidad razonable considerando que va a asociada fragmentaciones secundarias producto de la explosión de las vesículas expuestas. Así, el tamaño finito de un sistema introduce desviaciones sistemáticas del comportamiento macroscópico en transformaciones de segundo orden.

Teniendo en mente que los exponentes $\alpha$ y $\beta$ se refieren al coeficiente de intensidad de esfuerzos en una fractura, en partículas de tamaño diferente, es posible asociar cada término a un proceso físico diferente, si comparamos fracturas de igual tamaño. Así, el término $\gamma 2$, idéntico en los 3 casos, sería asociado a la tensión superficial causada por la fractura, pues hay que recortar igual número de enlaces. El término en $\gamma 4$ estaría asociado a la energía elástica requerida para separar las dos superficies que se crearon al romper los enlaces. La diferencia en su coeficiente puede deberse a la diferencia de tamaño de las partículas en las que se aplica. El exponente $\alpha$ está asociado a partículas más pequeñas que pueden deformase más fácilmente, por lo que requiere una menor energía para formar las vesículas, lo que se reflejaría en un coeficiente de $3 / 4$. Para $\beta$, las partículas son más grandes y la fractura no cambia su volumen al no ceder sus paredes exteriores, lo que se refleja en un coeficiente mayor. El término $\gamma^{3}$ es una energía extra asociable a la presencia de vesículas.

\section{ECUACIÓN DE GRIFFITH}

La conservación de la energía incluida en el modelo de Landau, la dispersión $\gamma$ como resultado del análisis de procesos de fractura a nivel global, y la idea de estabilidad asociada al mínimo de la energía libre $\mathrm{G}$, sugieren analizar que las ecuaciones (5) a (7) puedan reflejar el modelo de Griffith para el crecimiento de fracturas.

Una derivación simple de ese modelo puede consultarse en Neal-sturgess (2008). En forma breve, Griffith propone que la energía total involucrada $(\mathrm{G}=\mathrm{U}$ - ST) puede considerarse como la suma de la energía de deformación, dada por $\mathrm{U}$ $=-\sigma^{2} \pi \mathrm{a}^{2} / 2 \mathrm{E}$, y la energía superficial asociada con la fractura, dada por $\mathrm{S}=2 \mathrm{a} \gamma_{0}$. Físicamente, "a" es el largo de la fractura; E el Módulo de Young del material; $\sigma$ el esfuerzo aplicado que causa la fractura en la partícula; y $\gamma_{\mathrm{o}}$ es la energía superficial asociada a la nueva superficie que se crea. La energía total $\mathrm{G}$ será entonces la suma de una energía positiva, debido a la creación de las superficies, y de una energía negativa de deformación, al permitirle a la zona cercana al extremo de la fractura liberar tensión.

Básicamente, Griffith propone que hay un valor $\mathrm{a}_{\mathrm{C}}$, obtenido a partir $\mathrm{de} \mathrm{dG} / \mathrm{da}=0$, tal que si $\mathrm{a}<\mathrm{a}_{\mathrm{C}}$ la fractura no crecerá, en tanto que la fractura seguirá creciendo una vez que se cumpla a $>$ $a_{C}$, pues el proceso de fractura generará la energía necesaria para auto-sostenerse.

Comparando con la ecuación (5):

$$
\mathrm{G}=2 \mathrm{a} \gamma_{\mathrm{o}}-\left(\sigma^{2} \pi \mathrm{a}^{2} / 2 \mathrm{E}\right)=\mathrm{Q}-\left[\gamma^{2} / 2-3 \gamma^{4} / 4\right]
$$

Se puede asociar el término lineal en a, con el término en $\gamma^{2}$; y el término en $\mathrm{a}^{2}$ asociado a la energía de deformación, con el término en $\gamma^{4}$ $=\left(\gamma^{2}\right)^{2}$. Básicamente se puede decir que son la misma ecuación expresada en dos variables diferentes, relacionadas por $\gamma^{2}=\mathrm{k}$ a, donde $\mathrm{k}$ es una constante de dimensión de longitud inversa. Así, la incorporación del modelo de Landau de transformaciones de segundo orden a la formulación hecha por Balankin, permite recuperar la relación de Griffith, permitiendo expresar k y Q en términos de los parámetros físicos de la tensión 
superficial $\gamma_{0}$, el módulo de Young E y la tensión externa $\sigma$ a que está expuesto el material.

Definiendo $\mathrm{k}_{\mathrm{o}}=\sigma^{2} \pi / 2 \mathrm{E} \gamma_{\mathrm{o}}$ y $\mathrm{Q}_{\mathrm{o}}=8 \mathrm{E} \gamma_{0}{ }^{2} / \sigma^{2} \pi$ se encuentra Q- $=3 \mathrm{Q}_{\mathrm{o}}$ y $\mathrm{Q}_{\beta}=4 \mathrm{Q}_{\mathrm{o}}$

Similarmente $\mathrm{k}-=\mathrm{k}_{\mathrm{o}} / 3 \mathrm{y} \mathrm{k}_{\beta}=\mathrm{k}_{\mathrm{o}} / 4$. En lo que sigue usaremos $\mathrm{Q}-=\mathrm{Q}+\mathrm{y} \mathrm{k}-=\mathrm{k}+$ en el supuesto de que el efecto de las vesículas se toma en cuenta vía el término $\gamma^{3}$.

En el ámbito $-0,57<\gamma<0$, asociado a la creación de fracturas en un material quebradizo, se espera que entre más tortuosa sea la superficie fracturada mayor será la energía requerida para su creación, en acuerdo con la forma de la energía de Gibbs obtenida.

\section{RELACIÓN DE GIFFITH CON BALANKIN}

Aplicando la regla de la cadena podemos escribir $\mathrm{dG} / \mathrm{d} \gamma=\left(\mathrm{dG} / \mathrm{d} \gamma^{2}\right)\left(\mathrm{d} \gamma^{2} / \mathrm{d} \gamma\right)$, y recordando que $\mathrm{d} \gamma^{2} / \mathrm{d} \gamma=2 \gamma$, vemos que el término $\mathrm{Q}\left[1-3 \gamma^{2}\right]$ es el relacionado con $\left(\mathrm{dG} / \mathrm{d} \gamma^{2}\right)$ y eventualmente con Griffith pues, como $\gamma^{2}=\mathrm{k}$ a, la derivada se vuelve $\mathrm{dG} / \mathrm{da}$.

Por ende, para el caso $\alpha$ - se tendrá $\mathrm{dF} / \mathrm{da}=$ $\mathrm{Q}\left[1-3 \gamma^{2}\right]=\mathrm{Q}[1-3 \mathrm{k}-\mathrm{aC}]=\mathrm{Q}\left[1-\mathrm{k}_{\mathrm{o}} \mathrm{aC}\right]=$ 0 , o sea, $k o=\mathrm{aC}^{-1}$. Para el caso $\beta$. se tendrá $\mathrm{dF} /$ $\mathrm{da}=\mathrm{Q}\left[1-4 \gamma^{2}\right]=\mathrm{Q}\left[1-4 \mathrm{k}_{\beta} \mathrm{a}_{\mathrm{C}}\right]=\mathrm{Q}\left[1-\mathrm{k}_{\mathrm{o}} \mathrm{a}_{\mathrm{C}}\right]=$ 0 , o sea, $\mathrm{k}_{\mathrm{o}}=\mathrm{a}_{\mathrm{C}}{ }^{-1}$. Este es un resultado esperable por que partimos de que el proceso de fractura es fractal, y por tanto no puede diferenciar entre una partícula pequeña (caso $\alpha$ ) y una partícula más grande (caso $\beta$ ). Para el caso $\alpha$, obtenemos Q$=3 Q_{0}=3^{2} a_{C} \gamma_{0}$ y para el caso $\beta: Q_{\beta}=4 Q_{o}=4^{2} a_{C} \gamma_{0}$. Por la definición de ko se ve que se puede definir un stress crítico $\sigma_{\mathrm{C}}$ tal que $\sigma_{\mathrm{C}}{ }^{2}=\mathrm{E} \gamma_{\mathrm{o}} / \pi \mathrm{a}_{\mathrm{C}}$ tal que si el stress externo $\sigma>\sigma_{\mathrm{C}}$ la fractura crecerá.

Además $\mathrm{dG} / \mathrm{da}=0$ corresponderá con el valor que hace $\left[1-3 \gamma^{2}\right]=0$, que es la condición que se discutió en Brenes (2014), y que corresponde a $\gamma_{\min }=-1 / \sqrt{ } 3$.

En cierta manera se ha cerrado el círculo, pues la definición inicial del exponente $\alpha=[1-$ $\mathrm{d}(1-\mathrm{H})] / 2 \mathrm{H}$ (Balankin, 1997), fue hecha a partir de la relación de Griffith.

La idea de relacionar la energía de fracturación con la rugosidad usando la dimensión fractal no es nueva (Williford, 1987), sin embargo no se ha hecho como parte de una teoría completa, como la que aquí se propone.

\section{INCORPORACIÓN DE LA TEMPERATURA}

El modelo de Landau propone que la temperatura se incluya por medio de la expresión $\tau=\left(\mathrm{T}-\mathrm{T}_{\mathrm{C}}\right) / \mathrm{T}_{\mathrm{C}}$, que multiplica exclusivamente al término cuadrático.

Las expresiones aproximadas aquí presentadas fueron deducidas a partir de expresiones muy características, producto a su vez de principios muy fundamentales. La dispersión $\gamma$ es producto de la conservación de la masa; las relaciones $\mathrm{D}=$ $-3 \gamma$ y $\mathrm{D}=3(1+\gamma)$ se originan de considerar la fragmentación como un proceso fractal; los exponentes que originan la energía de Gibbs son producto de la aplicación del concepto de auto-afinidad al proceso de fractura. Como resultado, la forma de la energía de Gibbs en función de la dispersión $\gamma$ queda determinada desde el inicio. La única manera, entonces, en que se puede lograr que $\mathrm{G}$ muestre un mínimo para $\gamma \neq 0$ para $\tau<0$, y un mínimo para $\gamma=0$ para el caso $\tau>0$ es incorporando la temperatura reducida $\tau$ como un coeficiente de toda la energía: $\mathrm{G} \rightarrow \tau \mathrm{G}$.

Las ecuaciones finales serán entonces

$$
\begin{aligned}
& \mathrm{G}_{-}=\mathrm{G}_{\mathrm{o}}+\mathrm{Q} \tau\left(\gamma^{2} / 2-3 \gamma^{4} / 4\right) \\
& \mathrm{G}_{+}=\mathrm{G}_{\mathrm{o}}+\mathrm{Q} \tau\left(\gamma^{2} / 2-3 \gamma^{4} / 4-\gamma^{3}\right) \\
& \mathrm{G}_{\beta}=\mathrm{G}_{\mathrm{o}}+\mathrm{Q} \tau\left(\gamma^{2} / 2-\gamma^{4}\right)
\end{aligned}
$$

La fase para $\mathrm{T}>\mathrm{T}_{\mathrm{C}}$ debe ser más simétrica, lo que sugiere asociar $\gamma=0$ (o sea, $\mathrm{H}=1$ ) a una fractura en un campo continuo, sin ningún carácter fractal. Aquí hay acuerdo con Balankin (1997) quien propone que $\mathrm{H}=1$ se asocie a una fractura auto-símil La deducción de los dos valores universales de $\mathrm{H}$ obtenidos en Brenes (2014) no queda afectada.

$\mathrm{G} \rightarrow \tau \mathrm{G}$ implica que tanto la ampliación de una fractura por rompimiento de los enlaces, como el reacomodo de las partículas dependen linealmente de la temperatura, y que de existir una temperatura crítica $T_{c}$, ésta estaría determinada 
por un proceso de fractura en el que a temperaturas mayores a Tc todos los fragmentos resultantes serían producto de fracturas con superficies que no mostrarán ningún carácter auto-afin. El modelo de Landau propone por simplicidad que el coeficiente del término $\gamma^{4}$ es constante, lo que no evita que en el caso de fracturas pueda también depender linealmente de $\tau$.

El modelo de Landau justifica la dependencia lineal en $\tau$ argumentando que la energía de Gibbs está definida como $\mathrm{G}=\mathrm{U}-\mathrm{TS}$, siendo $\mathrm{S}$ la entropía. Si se expande la entropía en términos de $\gamma$, con $\mathrm{dG} / \mathrm{d} \gamma=0$, se obtiene

$$
\mathrm{G}=\left(\mathrm{U}_{\mathrm{o}}-\mathrm{TS}_{\mathrm{o}}\right)-\left(\mathrm{E}_{2}-\mathrm{aT}\right) \gamma^{2}+\ldots=\mathrm{G}_{\mathrm{o}}+\mathrm{a}(\mathrm{T}-
$$
$\left.\mathrm{T}_{\mathrm{C}}\right) / \mathrm{T}_{\mathrm{C}}+\gamma^{2} \ldots=\mathrm{G}_{\mathrm{o}}+\mathrm{Q} \tau \mathrm{a} \gamma^{2} / 2 \ldots \ldots$

El término cuadrático, asociado a la tensión superficial, variará linealmente con la temperatura en acuerdo con lo que Eötvös encontró experimentalmente.

\section{Energía de Gibbs exacta}

Si se repite el análisis anterior partiendo de las fórmulas exactas, se obtienen las ecuaciones:

$$
\begin{aligned}
& \mathrm{G}_{\alpha-}=\mathrm{Q}\left(3 \tau \gamma^{2}-4 \gamma+4 \operatorname{Ln}(1+\gamma)-2 \gamma^{3}-9\right) \\
& \mathrm{G}_{\alpha+}=\mathrm{Q}\left(3 \tau \gamma^{2}+5 \gamma+5 \operatorname{Ln}(1-\gamma)+\gamma^{3}-9\right) \\
& \mathrm{G}_{\beta-}=\mathrm{Q}\left(3 \tau \gamma^{2}+3 \gamma+3 \operatorname{Ln}(1-\gamma)-6\right) \\
& \mathrm{G}_{\beta+}=\mathrm{Q}\left(3 \tau \gamma^{2}-3 \gamma+3 \operatorname{Ln}(1+\gamma)-6\right)
\end{aligned}
$$

Para su análisis utilizaremos los tres gráficos de las dos primeras ecuaciones, correspondientes al caso Alfa (Fig. 1). En la figura 1a se observa que para valores positivos de $\tau$ ( $\tau=1$, en este caso), se obtiene un mínimo local para $\gamma=0$, como lo propone Landau. Sin embargo, este mínimo se convierte en un máximo global a partir de los valores positivos de $\tau=0,846$ y $\tau=0,637$ (Fig. 1b), y no de valores negativos como lo propone Landau. Más aun, para valores de $\tau$ negativos (Fig. 1c), la energía de Gibbs muestra mínimos globales de $\mathrm{G}$ $\rightarrow-\infty$, cuando $\gamma \rightarrow \pm 1$. Tradicionalmente estos mínimos globales infinitos se interpretarían como un defecto de la teoría, pues la energía de Gibbs se espera que esté limitada por abajo. En el caso aquí estudiado, la dispersión $\gamma$ está controlada por un proceso de fragmentación secuencial que aporta la energía extra necesaria. De la distribución de Weibull obtenida por Brown (1989) se observa que $\gamma=-1$ está también asociada con un $\infty$, límite al que nunca se llega pues la fragmentación no puede continuar eternamente.

En la figura 2 se muestra el gráfico de las expresiones aproximadas de la energía de Gibbs para partículas pequeñas (caso Alfa). Claramente se aprecian dos mínimos globales con $\gamma \neq 0$, como lo requiere el modelo de Landau. Los valores de H universales deducidos según Brenes (2014) no se ven afectadas por corresponder a los casos en que los exponentes de la intensidad de esfuerzo deducidos por Balankin (1996) se anulan.

A lo largo del presente estudio se ha utilizado la dispersión $\gamma$ como el parámetro de orden. Sin embargo, el análisis puede también llevarse a cabo utilizando el coeficiente de rugosidad o de Hurst, $\mathrm{H}=1-\gamma$. Usando $\alpha=(\mathrm{dH}-\mathrm{d}+1) / 2 \mathrm{H}, \mathrm{y} \mathrm{D}=$ $3(1+\gamma)$ la ecuación (2) genera la figura 3 , en la que $\mathrm{dG} / \mathrm{d} \gamma=-\mathrm{dG} / \mathrm{dH}$ es expresada en función de $\mathrm{H}$.

Por analogía con la aplicación del Modelo de Landau a otros sistemas, puede analizarse si simultáneamente puede darse $\mathrm{G}=0, \mathrm{y} \mathrm{dG} / \mathrm{d} \gamma=0$ utilizando $\tau=\left(\mathrm{T}-\mathrm{T}_{\mathrm{o}}\right) / \mathrm{T}_{\mathrm{o}}$, donde temperatura $\mathrm{T}_{\mathrm{o}} \neq$ $\mathrm{T}_{\mathrm{C}}$. Se busca encontrar si el sistema analizado permite que la fase de baja simetría $(\mathrm{H}<1)$ pueda tener la misma energía que la fase más simétrica $(\mathrm{H}=1)$ y aun así estar en equilibrio $(\mathrm{dG} / \mathrm{d} \gamma=0)$.

En la figura 4 se observa que para fragmentaciones primarias en partículas pequeñas esto es posible para $\tau=5,5$ (caso Alfa, con dispersión negativa); para fragmentaciones secundarias en partículas pequeñas (Caso Alfa, con dispersión positiva) es posible con $\tau=6,3$, y para partículas más grandes (caso Beta) puede darse para $\tau=$ 4,2 . Los valores positivos de $\tau$ sugieren que se empezó con temperaturas bajas, en las que son posibles fracturas auto afines con $\gamma \neq 0(\mathrm{H}<1)$, y aumentándola hasta que la temperatura es lo suficientemente alta para que las partículas que componen el material puedan reacomodarse y dar origen a fracturas auto similares $(\gamma=0, \mathrm{H}=$ 1). La temperatura a la que eso sucede la asimilaremos a la temperatura de transición al vidrio $T_{g}$. En la figura 4 se nota igualmente que los valores 

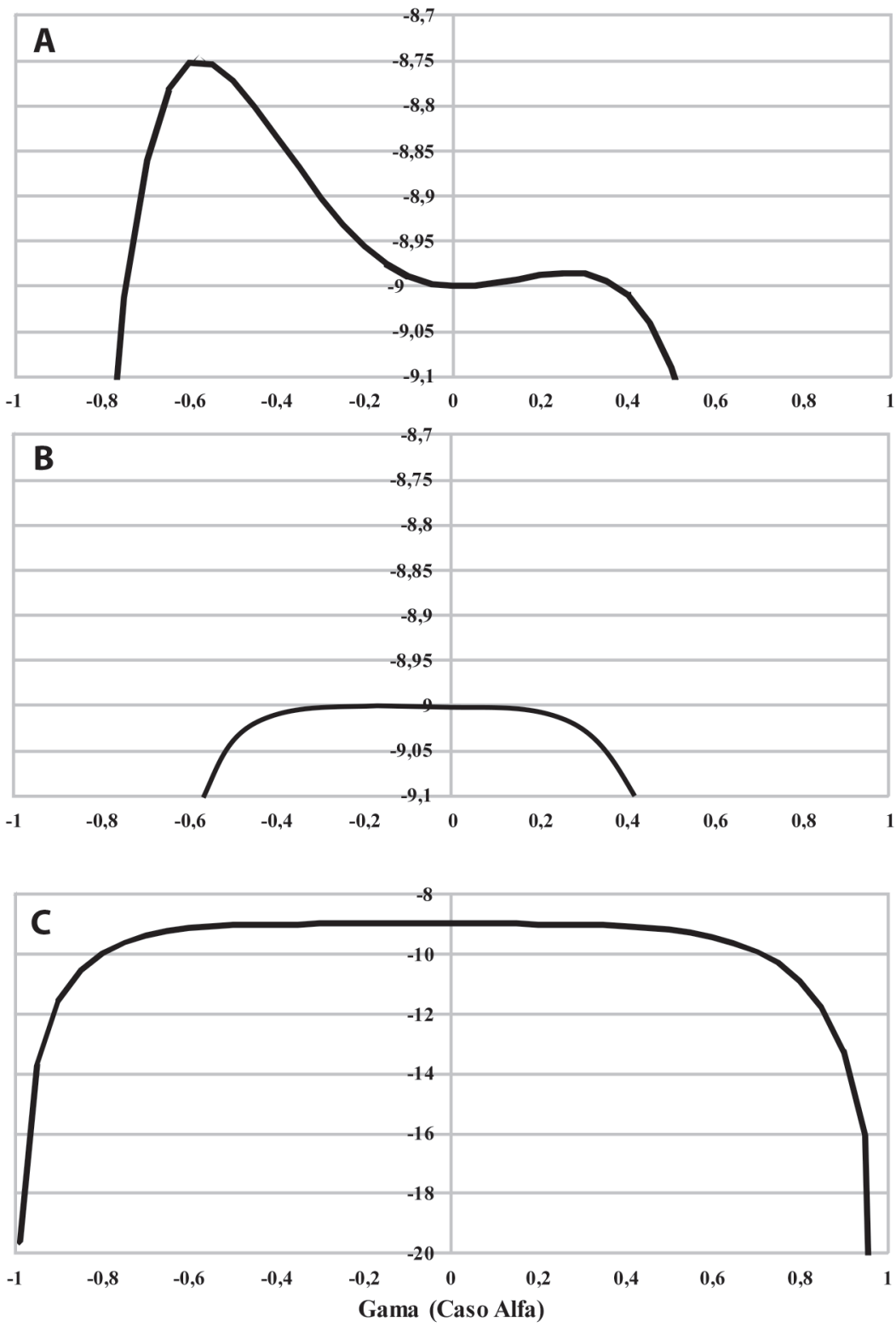

Fig. 1: Gráficos correspondientes al caso Alfa. A) Gráfico de la expresión exacta de la energía de Gibbs para partículas pequeñas, cuando $\mathrm{T}>\mathrm{Tc}$ mostrando un mínimo local en $\gamma=0$. Tau=1 para toda Gama. B) Gráfico de la expresión exacta de la energía de Gibbs para partículas pequeñas para los valores de $\tau$ en los cuales el mínimo local en $\gamma=0$ se torna un máximo, indicando una transformación de fase por rompimiento de simetría. Tau =0,846 (Gama $<0)$, Tau $=0,637(\mathrm{Gama}>0)$. C) Gráfico de la expresión exacta de la energía de Gibbs para partículas pequeñas para $\mathrm{T}<\mathrm{Tc}$ mostrando que la opción $\gamma=0$ es inestable, por ser un máximo local. Tau $=-1$ para todo Gama. 

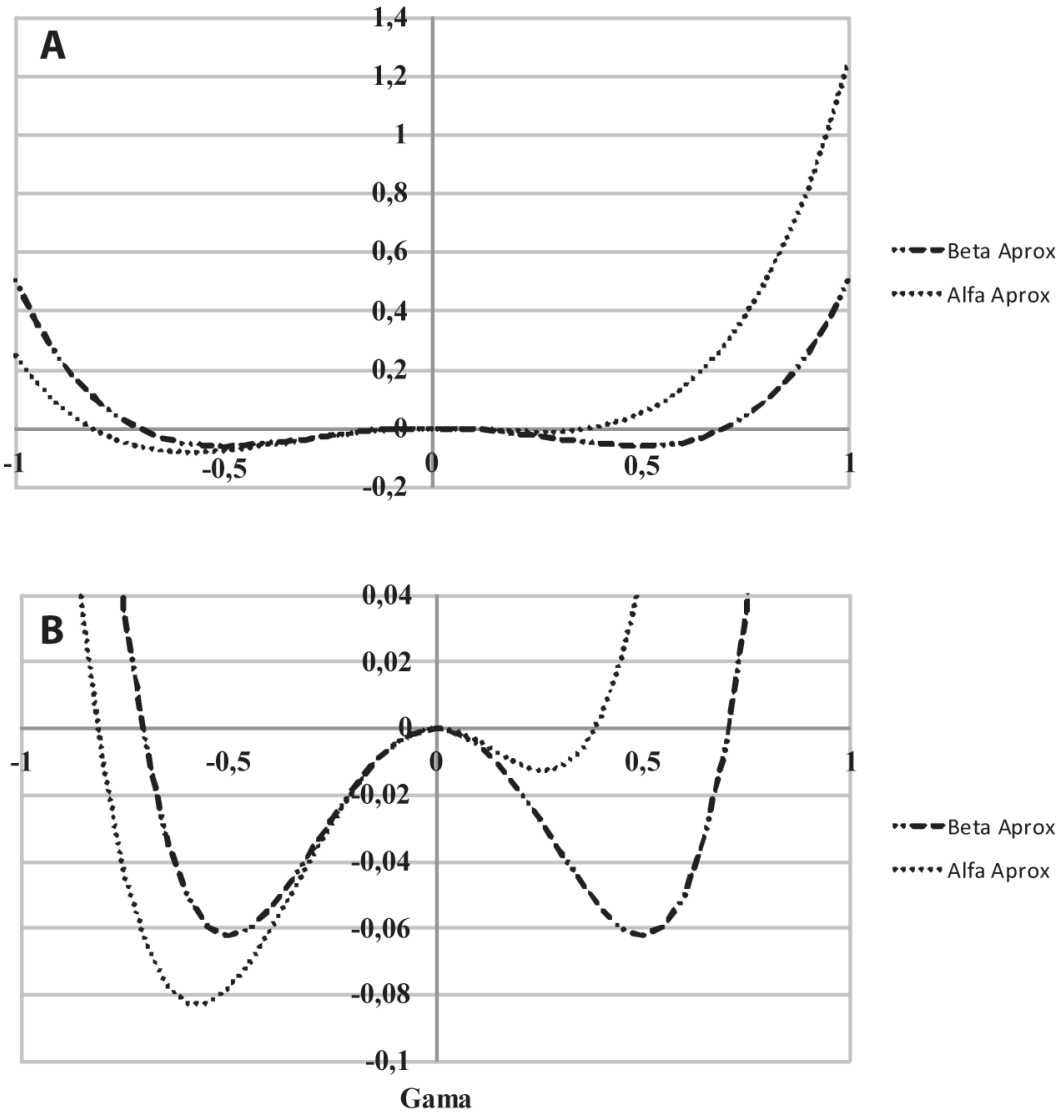

Fig. 2: Gráfico de las expresiones aproximadas de la energía de Gibbs para partículas pequeñas (caso Alfa). A) Gráfico de la energía de Gibbs aproximada para partículas pequeñas (caso Alfa), y partículas grandes (caso Beta). Nótese que la energía es finita para los casos $\gamma= \pm 1$. B) Ampliación de la figura 2a para mostrar mayor detalle, y permitir la comparación de ambos casos.

respectivos de $\gamma$ corresponden a estados metaestables, en los 3 casos.

La temperatura $T_{0}$ podría ser la deducida de la relación Vogel-Fulcher para viscosidad. Los valores obtenidos en este trabajo $\left(\mathrm{T}_{\mathrm{g}}=6,5 \mathrm{~T}_{\mathrm{o}} ; \mathrm{T}_{\mathrm{g}}=\right.$ $7,3 \mathrm{~T}_{\mathrm{o}}$ y $\mathrm{T}_{\mathrm{g}}=5,2 \mathrm{~T}_{\mathrm{o}}$ ) comparaban bien con el valor para $\mathrm{SiO} 2 \mathrm{de}_{\mathrm{g}}=5,4 \mathrm{~T}_{\mathrm{o}}$ (Sethna et al., 1991) y el $\mathrm{de}_{\mathrm{g}}=10,4 \mathrm{~T}_{\mathrm{o}}$ (Ferreira \& Aparicio, 2007).

Esta idea de que a mayor temperatura, las fluctuaciones serían más energéticas, pudiendo aumentar la rugosidad de la superficie fue propuesta hace varias décadas para películas por deposición (Burton, Cabrera \& Frank, 1951).

\section{DISCUSIÓN DE LOS RESULTADOS}

Las ecuaciones (5), (6) y (7) se basan en: a) los exponentes $\alpha$ y $\beta$, mayores o iguales a cero, derivados por Balankin para sólidos quebradizos, b) en la asociación de Brown (1989) a una frag-

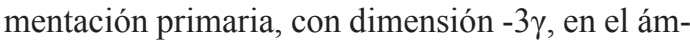
bito $(-1<\gamma<0)$, y c) a la asociación de Brenes \& Alvarado (2013) a fragmentaciones secundarias en el ámbito $(0<\gamma<1)$, con una dimensión 3(1+ $\gamma)$. Por ende, las ecuaciones (5), (6) y (7) tiene un carácter muy general al estar relacionadas con los principios fundamentales de conservación de 


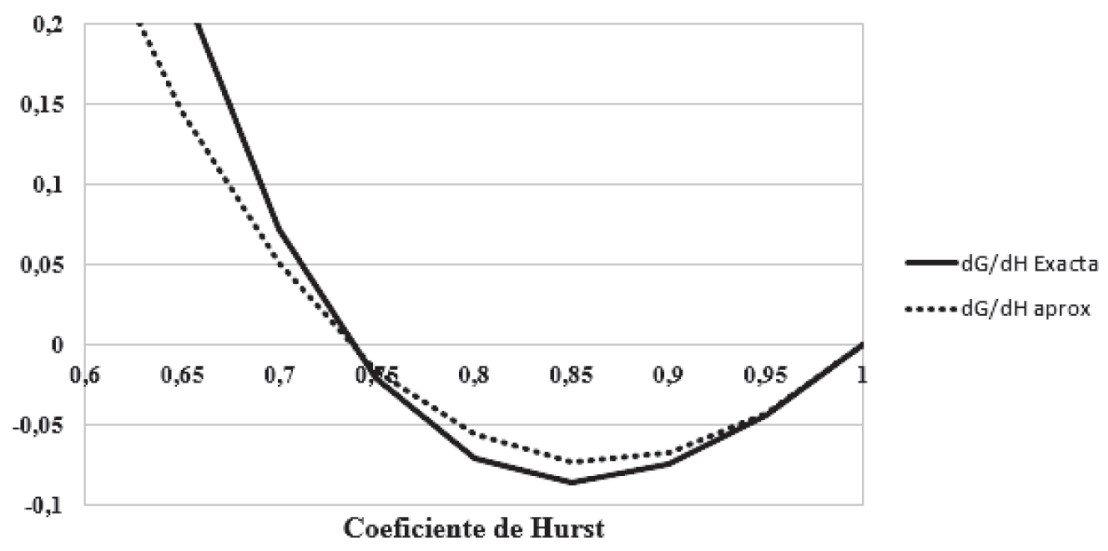

Fig. 3: Variación de la energía de Gibbs, comparación exacta versus aproximada (caso Alfa con dispersión positiva) con el coeficiente de rugosidad $\mathrm{H}$. Puede notarse que $\mathrm{G}$ varía directamente con $\mathrm{H}(\mathrm{dG} / \mathrm{dH}>0)$ en el ámbito $0,263 \leq \gamma \leq 1$, e inversamente con $\mathrm{H}(\mathrm{dG} / \mathrm{dH}<0)$ en el ámbito $0 \leq \gamma \leq 0,263$. El valor de $\gamma=0,263$ es uno de los valores universales analizados en Brenes (2014).

la masa, y de la energía, al punto que reproducen la ecuación de Griffith.

Con ayuda del coeficiente de Hurst $\mathrm{H}=1$ $|\gamma|$, y de la condición $1 / 2<\mathrm{H}<1$ para fracturas en materiales quebradizos (freatomagmáticos, según se corroboró con análisis en campo en el Irazú), se corroboraron los resultados del modelo de Landau para $0<\gamma<\gamma_{\min }$, donde $\mathrm{G}\left(\gamma_{\min }\right)$ es un mínimo local.

La fragmentación del magma puede verse como un aumento progresivo de un orden de corto alcance, debido a reordenamientos locales, que finalmente conducen a un ordenamiento de largo alcance con el resultado de una falla quebradiza de muchas vesículas prácticamente al mismo tiempo. Esto concuerda muy bien con la idea de que las transformaciones de fase son un efecto colectivo que depende de la interacción de las partículas.

Podría en este punto objetarse que la aproximación de Landau deja de ser válida bajo estas condiciones. $\mathrm{Lu}$ (2007), Lu et al (1995) y Bai et al. (1994) mostraron que la relación entre la rugosidad y la resistencia al impacto es exactamente opuesta para materiales quebradizos y dúctiles, relación similar a lo que se obtiene entre la energía de Gibbs y $\gamma$ obtenida por el modelo de Landau. Llamamos la atención al lector que Kuhn \& Müller (2011) desarrollaron la idea de un campo de fracturas que sigue una evolución del tipo Ginzburg-Landau, proceso que es tratado como un problema de transición de fases. La idea de aplicar el modelo de Landau a transiciones de vidrio ya fue utilizada por Goldstein (1980). En Sethna et al. (1991) explícitamente se indica que la transición del vidrio es una tradicional transición de segundo orden, con una componente estática y una dinámica.

Quizá estos resultados puedan entenderse como un mecanismo de Kibble-Zurek (Zurek \& Dorner, 2008) según el cual la transición de fase se da en el espacio, no en el tiempo. En el caso de las erupciones volcánicas, el magma en contacto con el aire en la parte superior sería el que tendría su temperatura cercana a la de transición del vidrio, en acuerdo con el "foam" que produce la ceniza. No se ahondará más en este punto, pues lo que aquí se presenta es un bosquejo de cómo se podrían unir todos estos mecanismos en una posible teoría coherente.

También podría objetarse que la dependencia lineal de la tensión superficial con la temperatura, específicamente con (T- $\mathrm{T}_{\mathrm{C}}$ ), propuesta por Eötvös, fue luego mejorada por Ramsay y Shields al encontrar experimentalmente, utilizando solventes orgánicos, la relación $\left(\mathrm{T}+6^{\circ} \mathrm{C}-\mathrm{T}_{\mathrm{C}}\right)$. El valor de $6^{\circ} \mathrm{C}$ es un valor promedio. Esta expresión sugiere la posibilidad de una tensión superficial negativa, lo que físicamente no puede ser. En el caso del mercurio, el ángulo de contacto es $<90^{\circ}$ debido a que se involucran 3 cuerpos: mercurio, aire, y vidrio, en lugar de una sola superficie: mercurio-aire. 

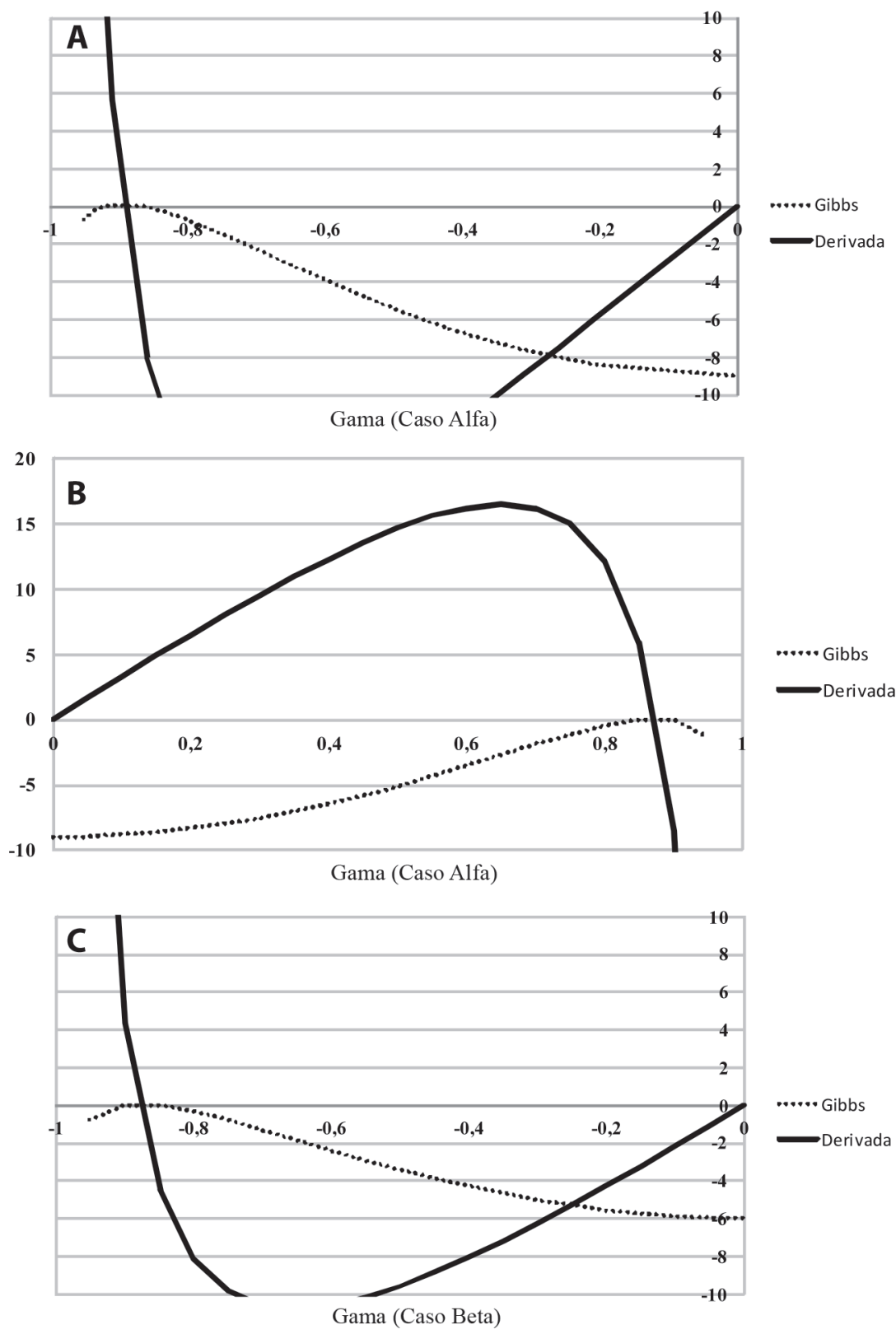

Fig. 4: Energía de Gibbs. A) Gráfico de la expresión exacta de la energía de Gibbs y su derivada (caso Alfa, dispersión negativa) que muestra toman el valor de cero cerca de $\gamma=-0,9$ cuando $\tau=5,5$. B) Gráfico de la expresión exacta de la energía de Gibbs y su derivada (caso Alfa, dispersión positiva) que muestra toman el valor de cero cerca de $\gamma=0,9$ cuando $\tau=6,3$. C) Gráfico de la expresión exacta de la energía de Gibbs y su derivada (caso Beta) que muestra toman el valor de cero cerca de $\gamma= \pm 0,9$ cuando $\tau=4,2$.

En el caso de que la corrección de Ramsay y Shields aplique también al magma, con un valor $\Delta$ diferente a $6^{\circ} \mathrm{C}$, se propone asociarlo a una "Transición del vidrio", con una temperatura de transición $\mathrm{T}_{\mathrm{g}}=\mathrm{T}_{\mathrm{C}}-\Delta$, donde $\mathrm{T}_{\mathrm{C}}$ seguiría siendo la temperatura en que se da la transformación de fase de Landau $\left(\mathrm{T}_{\mathrm{g}}<\mathrm{T}_{\mathrm{C}}\right)$. En el caso del magma, las paredes del conducto pueden constituir el necesario 
tercer cuerpo para no tener una tensión superficial negativa. Puede consultarse Barbero et al. (1991), Sparks (2003), y Gonnerman \& Manga (2003) para ahondar en este punto.

En el presente trabajo se entiende por "Transición del vidrio" aquella transición reversible de un material derretido que puede volverse un sólido quebradizo. El material presentará un cambio suave en el coeficiente de expansión térmica y en el calor específico. Según este planteamiento, la Transición del vidrio es parte de una transformación de segundo orden. Se debe advertir, no obstante, que hay propuestas de que sea una transformación de fase per se. (Angell et al., 2000; Ojovan, 2004).

Dingwell (1997) propone que en la región donde la deformación depende linealmente del esfuerzo, el magma es enteramente dúctil o viscoso comportándose como un líquido. Sin embargo, al comportarse como un sólido, es enteramente elástico si los esfuerzos son pequeños, o quebradizo si los esfuerzos son apreciables. En Tuffen et al. (2008) se analiza el caso del magma sufriendo fracturas en una manera similar a un sólido quebradizo.

Además, un magma vesicular se fragmentará cuando la tensión de tracción de las paredes interiores de las vesículas exceda la resistencia a la tracción del magma. Una visión más cuantitativa de la transición dúctil-quebradizo puede encontrarse en el trabajo clásico de Cottrell (1958).

Es importante destacar aquí que el comportamiento crítico de sistemas con interacciones de corto alcance se transforma en uno de campo medio (mean-field, como es el caso del modelo de Landau), cuando el efecto de las fluctuaciones del parámetro de orden pueden desestimarse. La expresión para $\mathrm{G}_{\alpha_{+}}$se asoció a un magma con vesículas que van interactuando entre ellas conforme el proceso eruptivo se desarrolla, constituyéndose así en las interacciones de corto alcance mencionadas. Los dos modelos propuestos para explicar las erupciones basálticas explosivas utilizan estas ideas: el modelo dependiente de la velocidad creciente (Wilson \& Head, 1981), y el del colapso de la espuma (foam) ( Jaupart \& Vergniolle, 1989; y Vergniolle \& Jaupart, 1990). Este segundo modelo se basa en la interacción del magma con las paredes de la cámara magmática y/o el conducto.

\section{CONCEPTO DE STRESS CRÍTICO EFECTIVO}

La dispersión $\gamma$ es una propiedad de una distribución por tamaño de partículas que sufrió una fragmentación secuencial (SFT). En el caso de fragmentación freatomagmática, se permiten fracturas primarias cuando se cumple $\gamma \geq \gamma_{\min }$. Sabiendo que k- $\mathrm{a}=\gamma^{2}$, se tiene $\mathrm{k}-\mathrm{a}_{\mathrm{C}}=\gamma_{\min }{ }^{2} \mathrm{y}$ $\mathrm{a}=\left(\gamma / \gamma_{\min }\right)^{2} \mathrm{a}_{\mathrm{C}}$, por lo que a $>\mathrm{a}_{\mathrm{C}}$. Por otra parte, Griffith propuso que cuando el stress externo $\sigma$ es mayor hay un stress crítico $\sigma_{\mathrm{C}}$, la fractura progresa. La unión de ambas ideas lleva a $\sigma>\sigma_{\mathrm{c}}$ $\left(\gamma_{\min } / \gamma\right)=\sigma_{C}^{1}$. El nuevo valor de stress crítico $\sigma_{C}^{1}$, diferente al que propuso Griffith, lo denominamos "stress crítico efectivo". Por tanto, la idea original de Griffith se mantiene si se redefine como que la fractura crecerá si el stress externo $\sigma$ es mayor que un stress crítico efectivo $\sigma^{1}$.

En el caso particular del magma, éste es un material muy complejo que en el proceso eruptivo puede variar su composición, temperatura, presión externa, sufrir exolución de los volátiles contenidos en sus vesículas, etc. Debido a este cambio de sus condiciones el stress crítico propuesto por Griffith variará, lo que puede llevar a que se cumpla que $\sigma_{\mathrm{C}}^{1}<\sigma<\sigma_{\mathrm{C}}$. Es decir, aun cuando el esfuerzo externo $\sigma$ no cambie, las condiciones que impedían que una fractura progresara $\left(\sigma<\sigma_{\mathrm{C}}\right)$ pueden haberse cambiando por agentes externos (adición de agua, por ejemplo), cambiando el $\sigma_{\mathrm{C}}$ original por $\sigma_{\mathrm{C}}^{1}$ permitiendo que las fracturas puedan entonces progresar $\left(\sigma^{1}{ }_{C}\right.$ $<\sigma$ ). De seguido analizaremos todas las posibles opciones que esta idea permite.

\section{ANÁLISIS DE TODAS LAS OPCIONES}

La incorporación de un stress efectivo, producto de la unión del modelo de Landau con el del modelo fractal de fractura, sugiere la existencia de otros posibles procesos. En lo que sigue se considerarán todas las opciones posibles, y se analizará la posibilidad de que algunos de ellos, sino todos, se den en la realidad. Para ello, enfatizaremos que la tensión superficial es un proceso de superficie, y que la energía de deformación lo es de volumen. 
Esta posición está muy de acuerdo con las ideas propuestas por Imry \& Ma (1975), al examinar la energía de las paredes que delimitan de un dominio de tamaño L. Para dimensiones $d \geq$ 2, al dividirse la partícula en dominios el sistema gana energía "volumétrica", y pierde energía "superficial". Este es básicamente el argumento de Griffith, visto de una manera más general. Imry $\&$ Ma (1975) argumentan que para $\mathrm{d} \leq 4$ siempre habrá un valor de L lo suficientemente grande que haga energéticamente más favorable un sistema dividido en dominios, aun cuando el campo externo a que esté sometido sea muy débil.

Para facilidad del lector, se han sistematizado los resultados obtenidos de estudiar todas las combinaciones de energía de superficie mayor o menor a la de volumen, en conjunto con las dos alternativas de $\gamma \geq \gamma_{\min }$ y $\gamma \leq \gamma_{\min }, \mathrm{y}$ de exponente $\alpha$ y $\beta$.

En el cuadro 1 se presentan los resultados para el caso $\gamma<0$. En el cuadro 2 se muestran los correspondientes resultados para $\gamma>0$. Dentro de cada cuadro la primera columna es producto de la división según si el efecto superficial es mayor o menor que el volumétrico, según se ha discutido en el texto. La columna siguiente es producto del valor relativo de la dispersión $\gamma$ respecto a $\gamma \mathrm{min}$. Debe tenerse presente que el valor de $\gamma$ min correspondiente para cada caso es diferente.

La columna siguiente muestra la posición relativa del stress crítico efectivo respecto al stress externo $\sigma$ y al stress crítico $\sigma \mathrm{C}$. Le sigue una columna con una brevísima descripción del tipo de fragmentación posible: magmática, si $0<\mathrm{H}<$ $1 / 2$, y freatomagmática si $1 / 2<\mathrm{H}<1$. El término "normal" indica que la existencia de un nuevo stress crítico no afecta el proceso; por "abortada" debe entenderse que el proceso se ve afectado negativamente, requiriéndose de un mayor stress externo para producirse. Inversamente, cuando el stress externo requerido es menor que el esperado se denominará "inducido".

A pesar de lo general de la deducción, el análisis de cada una de las posibilidades se hará con ejemplos relacionados con actividad volcánica. La similitud formal de los ejemplos con lo presentado en los cuadros, sugiere que pueden ser explicados refinando el modelo aquí presentado.
Las opciones (última columna) 1, 3, 4, 6, 7, 9,10 y 12 de los Cuadros 1 y 2 corresponden al criterio original de Griffith, pues el nuevo $\sigma 1 \mathrm{C}$ no lo modifica, por lo que se denominaron "normales". Las otras opciones se tratarán por separado.

\section{Opción 2}

Según esta opción, una fragmentación freatomagmática puede darse aún cuando el stress externo no haya alcanzado el valor crítico según Griffith. Una posible causa sería la presencia de agua que debilita pero no llega a romper los enlaces entre las moléculas, como sucede con el conocido proceso de Corrosión por esfuerzo.

\section{Opción 5}

Esta alternativa puede referirse a la interacción del magma con un fluido frio (el refrigerante) que tiene una temperatura de vaporización menor a la del magma. Para mayor información puede consultarse Wohletz $(1983,1986)$. Como lo reporta Corradini (1981), la presencia de gases no condensables o una alta presión externa pueden suprimir la explosión del vapor.

\section{Opción 8}

Un posible ejemplo puede ser el caso de la erupción del volcán Soufiere Hills (Isla Monserrat) de Agosto a Octubre, 1997. En ese período se dieron varios episodios de nucleación de cristal a una presión umbral de $\approx 7 \mathrm{MPa}$, dando lugar a una supersaturación volátil en el resto del magma, lo que forzó nuevas exoluciones, que aumentaron la viscosidad iniciando un comportamiento quebradizo y las subsecuentes fracturas en el sistema. Mayor información puede obtenerse en Gonnerman \& Manga (2003). Por su parte, Tuffen et al. (2003) se refieren a magma que es repetidamente fracturado y recocido.

La transformación, poco tiempo previo a la fractura, del magma dúctil en un magma quebradizo que permite se fragmente, explicaría el 
Cuadro 1

Posibles ordenamientos de las variables $\sigma_{C}^{1}, \sigma_{C}, \sigma$ (definidas en el texto) para el caso de dispersión $\gamma$ negativa

\begin{tabular}{|c|c|c|c|c|}
\hline & Gama & Esfuerzos & Tipo fragmentación & Opción \\
\hline \multirow{3}{*}{$\begin{array}{c}\text { Sup }<\text { Vol } \\
\mathrm{T}>\mathrm{T}_{\mathrm{C}}\end{array}$} & \multirow{2}{*}{$\gamma \geq \gamma \min$} & $\sigma_{\mathrm{C}}^{1}<\sigma_{\mathrm{C}}<\sigma$ & Freato, normal & 1 \\
\hline & & $\sigma_{\mathrm{C}}^{1}<\sigma<\sigma_{\mathrm{C}}$ & Freato, inducido & 2 \\
\hline & $\gamma \leq \gamma \min$ & $\sigma_{\mathrm{C}}<\sigma_{\mathrm{C}}^{1}<\sigma$ & Magma, normal & 3 \\
\hline \multirow{3}{*}{$\begin{array}{c}\text { Sup }>\text { Vol } \\
T<T_{C}\end{array}$} & $\gamma \geq \gamma \min$ & $\sigma<\sigma_{\mathrm{C}}^{1}<\sigma_{\mathrm{C}}$ & Freato, normal & 4 \\
\hline & \multirow{2}{*}{$\gamma \leq \gamma \min$} & $\sigma \mathrm{C}<\sigma<\sigma_{\mathrm{C}}^{1}$ & Magma, abortado & 5 \\
\hline & & $\sigma<\sigma_{\mathrm{C}}<\sigma_{\mathrm{C}}^{1}$ & Magma, normal & 6 \\
\hline
\end{tabular}

porque podemos utilizar para el caso de magma dúctil los mismos exponentes $\alpha$ y $\beta$ derivados por Balankin (1987) para material quebradizo. Nótese la similitud con las opciones 2 y 8 , y como en la primera interviene el agua, en tanto en la segunda intervienen las vesículas.

\section{Opción 11}

Se sugiere puede asociarse al concepto de materiales quasi-brittle, definidos por Irwin (1947) y Orowan (1950). Estos son materiales que bajo los test normales de tensión se comportan como altamente dúctiles, fracturándose de manera quebradiza una vez que las primeras fracturas aparecen.

Esto es lo que sucede con el trabajo en frío, en que una pieza de metal es repetidamente golpeada con un martillo, causando que un defecto se vaya trasladando, hasta llegar a un punto en que un obstáculo o le permite desplazarse más. Por lo general, ese obstáculo es otro defecto orientado $\mathrm{n}$ una dirección diferente, que se ha nucleado en otro plano. Eventualmente un grupo de dislocaciones convergen en una zona que requiere un mayor esfuerzo para romper el material (Kreidler \& Anderson, 1996).

Otro ejemplo es el del efecto Joffe, descrito en Barnes (1933), en que el agua aumenta la ductibilidad del material, y previene la formación y crecimiento de las fracturas. Así, una barra de cloruro de potasio se quebrará si se dobla en aire seco, pero se deformará un poco si el aire está húmedo, y si está sumergida en una solución acuosa se vuelve totalmente plástica. El agua impide el crecimiento de fracturas que se inician en la superficie. La velocidad con la que las fracturas se mueven en cristales haluro-alcalinos y óxido de magnesio, también es reducida por la presencia del agua. (Buckley, 1981).

Se reitera que los comentarios anteriores son solo posibilidades de cómo esta integración de SFT-Balankin-Landau pudiera arrojar luz sobre el proceso eruptivo. De darse esa posibilidad los valores de $\gamma$ obtenidos a partir de SFT aplicada a granulometrías permitirían obtener información sobre los diversos procesos que se dieron, de la misma manera en que ahora se usa para los fenómenos de transporte: balístico, saltación suspensión, etc.

\section{CONCLUSIONES}

El modelo aquí propuesto es la unión de varios resultados, que independientemente han mostrado su validez y utilidad. No obstante, puede cuestionarse si pueden unirse de la manera aquí propuesta. A continuación se presenta un grupo de ideas que, quizá, logren dar respuesta a algunos de esos interrogantes. Se recuerda al lector que este es un modelo en construcción, y que aún no se tiene a mano los datos experimentales requeridos para ponerlo a prueba.

Los exponentes $\alpha$ y $\beta$, propuestos por Balankin, fueron derivados para materiales quebradizos, asignándoseles un valor nulo para materiales dúctiles. 
Cuadro 2

Posibles ordenamientos de las variables $\sigma_{C}^{1}, \sigma_{C}, \sigma$ (definidas en el texto) para el caso de dispersión $\gamma$ positiva

\begin{tabular}{|c|c|c|c|c|}
\hline & Gama & Esfuerzos & Tipo fragmentación & Opción \\
\hline \multirow{3}{*}{$\begin{array}{c}\text { Sup }<\text { Vol } \\
\mathrm{T}>\mathrm{T}_{\mathrm{C}}\end{array}$} & \multirow{2}{*}{$\gamma \geq \gamma \min$} & $\sigma_{\mathrm{C}}^{1}<\sigma_{\mathrm{C}}<\sigma$ & Magma, normal & 7 \\
\hline & & $\sigma_{\mathrm{C}}^{1}<\sigma<\sigma_{\mathrm{C}}$ & Magma, inducido & 8 \\
\hline & $\gamma \leq \gamma \min$ & $\sigma_{\mathrm{C}}<\sigma_{\mathrm{C}}^{1}<\sigma$ & Freato, normal & 9 \\
\hline \multirow{3}{*}{$\begin{array}{c}\text { Sup }>\text { Vol } \\
\mathrm{T}<\mathrm{T}_{\mathrm{C}}\end{array}$} & $\gamma \geq \gamma \min$ & $\sigma<\sigma 1 \mathrm{C}<\sigma_{\mathrm{C}}$ & Magma, normal & 10 \\
\hline & \multirow{2}{*}{$\gamma \leq \gamma \min$} & $\sigma_{\mathrm{C}}<\sigma<\sigma_{\mathrm{C}}^{1}$ & Freato, abortado & 11 \\
\hline & & $\sigma<\sigma_{\mathrm{C}}<\sigma_{\mathrm{C}}^{1}$ & Freato, normal & 12 \\
\hline
\end{tabular}

La dispersión propuesta inicialmente por Brown (1989), y aplicada por Wohletz (1989) a procesos eruptivos, es transformada en un parámetro de orden del modelo de Landau para transformaciones de segundo orden, que es usualmente válido cerca del punto crítico $\mathrm{T} \approx \mathrm{T}_{\mathrm{C}}$. En el caso especial de materiales superconductores, sin embargo, la ecuación de Ginzburg-Landau mostró ser exacta en todo el ámbito $0<\mathrm{T} \leq \mathrm{T}_{\mathrm{C}}$, donde la temperatura $\mathrm{T}_{\mathrm{C}}$ está bien definida.

Dado el carácter de teoría de campo medio (mean field theory) del modelo de Landau, el proceso de la formación de partículas por fragmentación del magma, su posterior solidificación por enfriamiento, y eventual fractura se consideran como una sola unidad.

En el ámbito $\mathrm{T}_{\mathrm{g}}=\mathrm{T}_{\mathrm{C}}-\Delta<\mathrm{T}<\mathrm{T}_{\mathrm{C}}$, la curva de la energía aproximada de Gibbs versus dispersión se invierte, por el cambio de signo de $\tau=$ $\left(\mathrm{T}-\mathrm{T}_{\mathrm{C}}\right) / \mathrm{T}_{\mathrm{C}}$ sin que el ámbito de $\gamma$ definido por $\alpha$ y $\beta$ se vea afectado.

A pesar de su simplicidad, el modelo aquí propuesto permite sistematizar una serie de resultados: a) la energía aproximada de Gibbs versus el parámetro de orden tienen de manera natural la forma esperada por Landau; b) El rompimiento espontáneo de simetría predicho al variar la temperatura, en el caso de la energía aproximada de Gibbs, se comporta conforme lo esperado; c) la expresión exacta de G (ecuaciones 9a-9d) tiende a - $\infty$ conforme $\gamma \rightarrow-1$, en razón del logaritmo, en acuerdo con el límite deducido de la derivación de Brown de la distribución de Weibull, que se asocia a tamaños de partícula muy pequeños; d) por construcción el modelo permite asociar los dos valores universales de $\mathrm{H}$ a mínimos de energía; e) la expresión aproximada de la energía de Gibbs se asocia de manera natural con la expresión para fracturas de Griffith; f) las expresiones exactas de G tienen la misma forma general, variando el peso relativo de la energía elástica, lo que permite incluir la parte plástica de los extremos de las fracturas; g) la expresión exacta de la energía conduce naturalmente a proponer una transición de vidrio; h) el modelo permite proponer un nuevo stress crítico $\sigma_{\mathrm{C}}{ }^{1}$, diferente al $\sigma_{\mathrm{C}}$ que se deduce de Griffith, lo que sugiere la existencia de varios procesos, tales como corrosión por esfuerzo y dilatación, que se han encontrado en la realidad.

\section{AGRADECIMIENTOS}

El Dr. Guillermo Alvarado efectuó una exhaustiva revisión de la versión preliminar, sugiriendo cambios importantes. Los términos "abortado" e "inducido" utilizados en los cuadros 1 y 2 son de su autoría. Se agradece también a un árbitro anónimo por sus aportes.

\section{REFERENCIAS BIBLIOGRÁFICAS}

ANGELL, C. A., NGAI, K. L., MCKENNA, G. B., MCMILLAN, P. F., \& MARTIN, S. W.,2000: Relaxation in glassforming liquids and amorphous solids.- App. Phys. Rev. 88(6): 3113-3157. 
BAI, Y. L., LU, C., KE, F. J. \& XIE, M. F., 1994. Evolution induced catastrophe.- Phys. Lett. A, 185: 196-200.

BALANKIN, A., 1996: Mechanics of selfaffine cracks: the concept of equivalent traction, path integrals and energy release rate.- Rev. Mexicana de Física, 42(2): 161-171.

BALANKIN, A., 1997: Physics of fracture and mechanics of self-affine cracks.- Eng. Frac. Mech. 57: 135-203.

BARBERO, G., GABBASOVA, Z. \& MIRALDI, E., 1991- On the surface tension behavior near the critical temperature.- Mod. Phys. Lett. B5: 753. DOI: 10.1142/ S0217984991000939.

BARNES, R. B. 1933: The Plasticity of Rocksalt and Its Dependence upon Water.- Phys. Rev. 44: 898. DOI http://dx.doi.org/10.1103/ PhysRev.44.898.

BRENES, J., 2013: Aplicación de la teoría de fragmentación/transporte secuencial a los depósitos de las erupciones 1723 y $1963-$ 65 del Irazú, Costa Rica. Caso dispersión negativa.- Rev. Geol. Amér. Central, 48: 63-85.

BRENES, J., 2014: Aplicación del modelo de fractura fractal de Balankin al modelo fractal de actividad volcánica.- Rev. Geol. Amér. Central, 50: 83-98.

BRENES, J., \& ALVARADO, G. E., 2013: Aplicación de la teoría de fragmentación/ transporte secuencial a los depósitos de las erupciones 1723 y 1963-65 del Irazú, Costa Rica. Caso dispersión positive y modelo fractal.- Rev. Geol. Amér. Central, 48: 87-98.

BROWN, W. K., 1989: A theory of sequential fragmentation and its astronomical applications.- J. Astrophys. Astr. 10: 89-112.
BUCKLEY, D. 1981: Surface effects in adhesion, friction, wear, and lubrication.- 630 pags. Elsevier Scientific Publishing Company, The Netherlands.

BURTON, W. K., CABRERA, N. \& FRANK, F. C., 1951: The Growth of Crystals and the Equilibrium Structure of their Surfaces.Phil. Trans. R. Soc., 243: 299-358.

CORRADINI, M. L., 1981: Phenomenological modelling of the triggering phase of smallscale steam explosions experiments.- Nucl. Sci. Eng. 78: 154-170.

COTTRELL, A. H., 1958: Theory of brittle facture in steel and similar metals.- Trans. Metallurgical Soc. AIME, 212:192-203.

DEBENEDETTI，P. G.; STILLINGER，2001: Supercooled liquids and the glass transition.- Nature, 410(6825): 259-267.

DINGWELL, D., 1997: The Brittle-Ductile Transition in High-Level Granitic Magmas: Material Constraints.- J. Petrolog. 38(12): 1635-1644.

FERREIRA M. L. \& APARICIO, C. 2007- Data classification with the Vogel-Fulcher-TammannHesse viscosity equation using correspondence analysis.- Physica B398: 71-77.

GOLDSTEIN, M. 1980: Thermodynamic aspects of the glass transition: The applicability of the Landau theory of second-order transitions.- J. Macromolecular Sci. PartB: Physics, 18: 445-452.

GONNERMANN, H. M. \& MANGA, M., 2003: Explosive volcanism may not be an inevitable consequence of magma fragmentation.- Nature, 426: 432-435.

GUARINO, A., GARCIMART, A., \& CILIBERTO, S., 1998: An experimental test of the critical behavior of fracture precursors.- Eur. Phys. J. B6: 13-24. 
IMRY, Y. \& MA, S., 1975: Random-Field Instability of the Ordered State of Continuous Symmetry.- Phys. Rev. Lett. 35: 1399-1401

IRWIN, G., 1947: Fracture Dynamics. Fracturing of Metals.- Book of papers presented at the seminar on the fracturing of metals held during the Twenty-Ninth National Metal Congress and Exposion. Chicago 18-24 October. Cleveland, Ohio: $147-166$

JAUPART, C. \& VERGNIOLLE, S. 1989: The generation and collapse of a foam layer at the roof of a basaltic magma chamber.- J. Fluid Mech., 203: 347-380.

KREIDLER, E. R. \& ANDERSON, P. M., 1996: Orowan-based deformation model for layered metallic materials.- Mat. Res. Soc. Symp. Proc. 434: 159-170.

KUHN, C. \& MÜLLER, R., 2011: On an Energetic Interpretation of a Phase Field Model for Fracture.- PAMM. Proc. Appl. Math. Mech. 11: 159-160, DOI: 10.1002/ pamm.201110071.

LANDAU, L.D. \& LIFSCHITZ, E.M. 1988: Física Estadística, vol. 5 del Curso de Física Teórica. Editorial Reverté, Barcelona.

LU, C., 2007. Some notes on the study of fractals in fracture. In Proceedings of 5th Australasian Congress on Applied Mechanics, ACAM 2007, Brisbane, Australia: 234-239.

LU, C.; KE, F. J.; BAI, Y. L. \& XIA, M. F., 1995. Numerical simulation of evolution induced catastrophe, Sci. China Ser. A 38: 462-471.
NEAL-STURGESS, C. E., 2008: A Direct Derivation of the Griffith-Irwin Relationship using a Crack tip Unloading Stress Wave Model.- DOI: arXiv 0810.2218.

OJOVAN, M. I., 2004: Glass formation in amorphous $\mathrm{SiO}_{2}$ as a percolation phase transition in a system of network defects.- J. Experimental and Theoretical Physics, Letters, 79(12): 632-634.

OROWAN, E., 1950: Fundamentals of brittle behavior in metals. En: W. M. Murray \& J. C. Hunsaker (eds.), Fatique and Fracture of Metals, Book of papers presented at the Symposium held at the Massuchusetts Institute of Technology. Massachusetts, 19 - 22 June.The Technology press of the Massachusetts Institute of Technology and John Wiley \& Sons, Inc., New York: 139-167.

SANTAOJA, K., 1992: Basics of "Griffith's fracture mechanics".- Rakenteiden Mekaniikka, 25(3): 3-23.

SETHNA, J. , SHORE, J. \& HUANG, M., 1991: Scaling theory for the glass transition.Phys. Rev. B, 44: 4943- 4959.

SPARKS, R. S. J., 1978- The dynamics of bubble formation and growth in magmas: A review and analysis.- J. Volcan. Geotherm. Res. 3 : 1-37.

SPARKS, R. S. J., 2003: Dynamics of magma degassing.- Geol. Soc. London, Spec. Pub. 213: 5-22, DOI: $10.1144 / \mathrm{GSL}$. SP.2003.213.01.02

TUFFEN, H., DINGWELL, D. B., PINKERTON, H., 2003: Repeated fracture and healing 
of silicic magma generate flow banding and earthquakes?.- Geology, 31(12): 1089-1092.

TUFFEN, H., SMITH, R. \& SAMMONDS, P. R. 2008: Evidence for seismogenic fracture of silicic magma.- Nature, 453: 511-514.

VERGNIOLLE, S. \& JAUPART, C. 1990: Dynamics o degassing at Klauea Volcano, Hawaii.- J. Geophys. Res., 101: 20 433- 20447.

WILLIFORD, R. E. 1987. A Similarity Analysis of Fracture.- Proceedings of the December 1987 Winter Annual Meeting, ASME, Boston: 39-44.

WILSON, L. \& HEAD, J. W. 1981: Ascent and eruption of basaltic magma on the Earth and Moon.- J. Geophys. Res. 86: 2971-3001.

WOHLETZ, K., 1983: Mechanisms of hydrovolcanic pyroclast formation: grain-size, scanning electronic microscopy and experimental estudies.- J. Volcan. Geother. Res. 17: 31-63.

WOHLETZ, K. H., 1986, Explosive magma-water interactions: Thermodynamics, explosion mechanisms, and field studies.- Bull. Volcanol. 48: 245-264.

WOHLETZ, K. H., SHERIDAN, M. F. \& BROWN, K., 1989: Particle size distribution and the sequential fragmentation/transport theory applied to volcanic ash.- J. Geophys. Res. 94: 15,70315,721 .

ZHANG, Y. X., 1999: A criterion for the fragmentation of bubbly magma based on brittle failure theory.- Nature, 402: 648-650.

ZUREK, W. H. \& DORNER, U., 2008: Phase transition in space: how far does a symmetry bend before it breaks?.- Phil. Trans. R. Soc. A366: 2953-2972, DOI:10.1098/ rsta.2008.0069. 